\title{
A Hybrid Method for Signalling Transport in GMPLS Control Plane
}

\author{
Mirjana D. Stojanovic ${ }^{1}$, Suzana D. Miladic ${ }^{1,2}$, Goran Z. Markovic ${ }^{1}$ \\ ${ }^{I}$ Faculty of Transport and Traffic Engineering, University of Belgrade, \\ Vojvode Stepe 305, 11000 Belgrade, Serbia \\ ${ }^{2}$ Faculty of Transport and Traffic Engineering, University of East Sarajevo \\ Vojvode Misica 52, 74000 Doboj, Bosnia and Herzegovina \\ m.stojanovic@sf.bg.ac.rs
}

\begin{abstract}
This paper addresses the issue of signalling transport in control plane of the Generalized Multi-Protocol Label Switching (GMPLS) network. The two basic methods for establishing control channels in optical networks are the infibre-out-of-band and the out-of-fibre. Starting from the analysis of benefits and drawbacks of those methods, we propose and investigate a novel, flexible method for signalling transport, which is based on the analysis of the given network topology and combines the basic methods to achieve a trade-off between requirements for low latency and high reliability. Performance analysis has shown that, with hybrid method, data path setup times are close to the ones achieved with the in-fibreout-of-band method, while preserving satisfying reliability and flexibility to integrate into multi-domain networks.
\end{abstract}

Index Terms-Control plane; optical network; signalling; switching.

\section{INTRODUCTION}

Generalized Multi-Protocol Label Switching (GMPLS) extends basic MPLS concepts, which have been developed for packet switched networks, to make them applicable to systems with time division multiplexing, layer-2 switching, wavelength switching, and fibre switching. Separation of GMPLS control and data planes assumes appropriate signalling and routing protocols to enable optimal resource usage, the need for link management protocol, and advanced possibilities for traffic engineering in optical networks.

Signalling in control plane is needed to establish, maintain, modify, and terminate label switched paths (LSPs) in the data plane. LSP is a path through the network formed of cross-connected labels (resources) on a series of data plane links. Signalling messages are exchanged between software agents called signalling controllers. The signalling controller may be physically separated from the data switch, with a management protocol used to communicate with the switch [1]. Signalling controllers are identified by the Internet Protocol (IP) addresses; hence, control plane in GMPLS network is in fact an IP network.

Resource Reservation Protocol - Traffic Engineering (RSVP-TE) has been adopted and extended for signalling in

Manuscript received 18 October, 2015; accepted 30 April, 2016.

This research was partially funded by a grant (No. TR 32025) from the Ministry of Education, Science and Technological Development of Serbia.
GMPLS networks [2]. RSVP-TE messages are encapsulated in IP packets exchanged between signalling controllers. LSPs in GMPLS networks are typically bidirectional, as opposed to traditional MPLS LSPs. Bandwidth reservations can be asymmetric for the two path directions [3].

Signalling requirements mainly refer to low latency and high reliability. Since GMPLS signalling is separated from data and carried through IP protocol, these requirements are opposed to each other, and proper design of control plane is a challenging issue for network developers. This paper proposes and investigates a hybrid method for GMPLS signalling transport in optical networks, which aims to achieve low delays, while preserving satisfying network reliability.

The rest of the paper is organized as follows. Section II surveys the related work. Proposal of the hybrid signalling transport method is described in Section III. Performance analysis is presented in Section IV. Applicability of the proposed method to multi-domain scenarios has been discussed in Section V. Section VI concludes the paper.

\section{RELATED WORK}

GMPLS control channel is a physical or logical link between signalling controllers responsible for adjacent data switches. In optical networks, there are two basic methods for establishing control channels, namely the In-Fibre-Outof-Band (IFOB) and the Out-of-Fibre (OF) [1].

The IFOB method assumes assignment of a specific data channel for the signalling traffic, e.g., a specific timeslot, or a dedicated wavelength. Such a channel is called optical supervisory channel (OSC), and it must be terminated at each data switch. Although separated from the data traffic, signalling messages are carried over the same physical paths; hence, the IFOB configuration typically represents a symmetrical topology [4], [5].

The OF method assumes that the control channel utilizes a physical connection that follows a different physical path from the data path, and may be routed through a completely separate network. A single signalling controller may manage more than one data switch. The out-of-fibre configuration allows the deployment of asymmetrical topologies [4], [5].

Veeraraghavan and $\mathrm{Li}$ have shown that with hardwareaccelerated signalling engines, the IFOB method is a better 
option to keep end-to-end LSP setup time low [6].

On the other side, one of the main benefits of the OF method is that control channels remain alive upon data plane failures. Therefore, the Link Management Protocol (LMP) may take a simple fault localization procedure. With the IFOB method, fault localization usually relies on hardwarebased solutions.

Perelló et al. have compared fault localization times with the LMP and a hardware-based solution (the pilot tone-based procedure) [4]. Although LMP detection and localization times are generally higher than the ones achieved by the optical pilot-tone based procedure, their contribution to the total protection time (e.g., $50 \mathrm{~ms}$ ) could be assumed as minor even in large network topologies.

If OF method is applied, the control channel should be protected against individual link failures within the control plane [1]. Ruiz et al. propose the design of GMPLS control plane with resilience guarantees, where two parameters are introduced: the probability that LSP setup or teardown is dropped due to control plane link failure and control plane link failure recovery time [5].

Besides, the OF configuration can provide more flexible solutions, e.g., by introducing path computation elements (PCEs) to solve some of the limitations of scalability and visibility that affect control plane path routing [7].

Signalling requirements for end-to-end unidirectional service establishment in IP networks have been investigated in [8]. Analytical expressions for signalling traffic intensity, response times, and processor load have been derived for networks with star, ring, linear and full mesh topologies.

Our goal is to propose a novel, hybrid method for signalling transport, which combines the benefits of IFOB and OF methods jointly with the analysis of considered network topology to achieve a trade-off between the requirements for low latency and high reliability.

\section{A HYBrid SignALLING TRANSPORT MethoD}

The network is observed as an undirected graph with $N$ nodes and $E$ edges (links). Let $k_{n}$ be the node $n$ degree, which denotes number of node $n$ links with adjacent nodes. Hence, the average node degree $\bar{k}$ is defined as follows

$$
\bar{k}=\frac{1}{N} \sum_{n=1}^{N} k_{n}=\frac{2 E}{N} .
$$

Joint degree distribution, $J D D$, is the probability that a randomly selected edge connects $k$ - and $k^{\prime}$-degree nodes

$$
J D D\left(k, k^{\prime}\right)=E\left(k, k^{\prime}\right) / E,
$$

where $E\left(k, k^{\prime}\right)$ is the total number of edges connecting nodes of degrees $k$ and $k^{\prime}$.

The main idea of our proposal is that higher degree nodes should have built-in signalling controllers, while other nodes make signalling through proxy routers, which operate as separate controllers. In other words, the IFOB method, which assures reduced delay impairment, is applied only on the links that connect higher degree nodes. If such a link fails, in the worst case both data and control traffic will be lost. Certainly, fast recovery procedure should be activated either by some protection or restoration scheme [1]. To improve the data plane reliability, the percentage of such links should be limited. In the network segment where the OF method is applied, control channel should be protected by additional mechanisms against individual failures within the control plane (IP layer rerouting, MPLS fast rerouting).

Let $k_{\min }$ and $k_{\max }$ be the minimum and maximum node degrees, respectively, and $k_{\mathrm{T}}$ is the node degree threshold, $k_{\min }<k_{\mathrm{T}}<k_{\max }$. Further, $p_{\mathrm{IF}}$ is the probability that a randomly selected link connects nodes with $k_{n} \geq k_{\mathrm{T}}$, i.e.

$$
p_{\mathrm{IF}}=J D D\left(k_{n} \geq k_{\mathrm{T}}, k_{n} \geq k_{\mathrm{T}}\right)
$$

The predefined upper bound for $p_{\mathrm{IF}}$ is $\mathrm{P}_{\max }$. Our proposed hybrid signalling transport method is performed through the following steps:

Step 1: Set $\mathrm{P}_{\max }$;

Step 2: For each node $n, n=1,2, \ldots, N$ : Calculate node degree $k_{n}$;

Step 3: Find $k_{\min }$ and $k_{\max }$;

Step 4: Set threshold $k_{\mathrm{T}}, k_{\min }<k_{\mathrm{T}}<k_{\max }$;

Step 5: Calculate $p_{\mathrm{IF}}$;

Step 6: If $p_{\text {IF }} \leq \mathrm{P}_{\max }$ go to Step 7;

Else go to Step 4;

Step 7: For each node $n, n=1,2, \ldots, N$ :

If $k_{n} \geq k_{\mathrm{T}}$

Place signalling controller at optical switch; Else use proxy router.

The threshold $k_{\mathrm{T}}$ enables flexible planning and design of control plane. It may be a good practice in the planning process to set the initial value, e.g., $k_{\mathrm{T}}=\lceil\bar{k}\rceil$ and to further vary $k_{\mathrm{T}}$ to obtain the preferred control plane design.

Specification of proxy routers should depend on the network topology. We propose the following procedure. For each node $n$ with $k_{n}<k_{\mathrm{T}}$ adjacent nodes should be checked. If all neighbours are the nodes with $k \geq k_{\mathrm{T}}$, i.e., with built-in signalling controllers, proxy router should be added to the node $n$. Otherwise, a single proxy router may be shared among two or more adjacent nodes.

Application of the proposed method is depicted in Fig. 1.
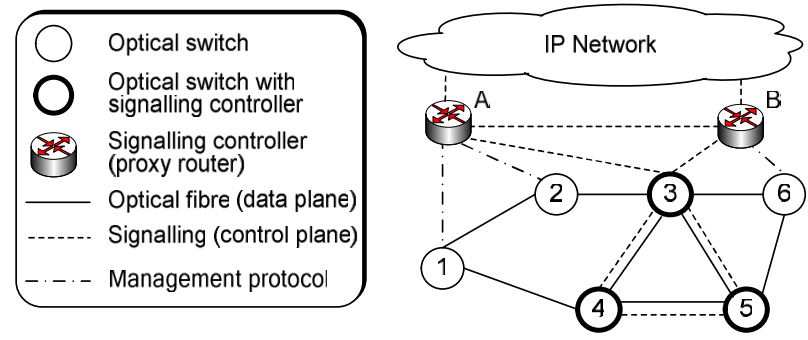

Fig. 1. Illustration of the proposed hybrid signalling transport method.

The example network topology consists of six nodes with 
the following degrees: $k_{1}=k_{2}=k_{6}=2, \quad k_{3}=4$, $k_{4}=k_{5}=3$. The average node degree is calculated according to (1) as $\bar{k}=2.67$. We further select the threshold $k_{\mathrm{T}}=\lceil\bar{k}\rceil=3$. Let $\mathrm{P}_{\max }$ be 0.5 . According to (2) we obtain $p_{\mathrm{IF}}=0.375<\mathrm{P}_{\max }$. Therefore, signalling controllers should be placed in switches 3,4 and 5. Adjacent nodes 1 and 2 have degrees less than $k_{\mathrm{T}}$, and share the same proxy router A. Neighbours of node 6 have built-in signalling controllers, so this node uses separate proxy router B. Proxy routers communicate with their associated data switches through a management protocol.

Figure 2 shows an example of RSVP-TE signalling during establishment of LSP 1-4-5-6 in the data plane.

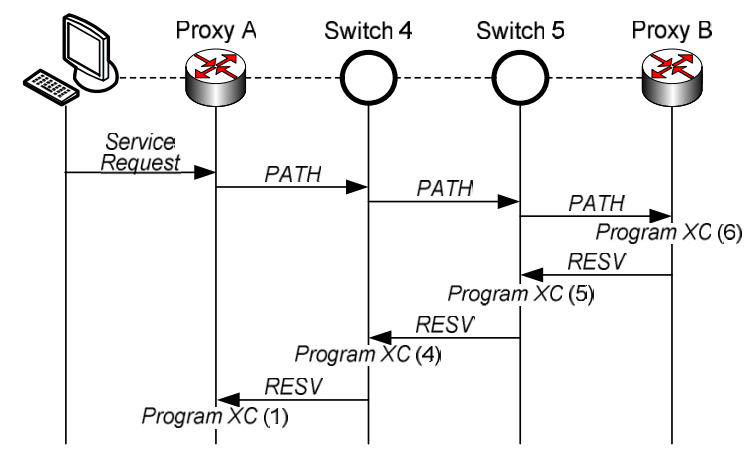

Fig. 2. Example of LSP setup (LSP 1-4-5-6, network from Fig. 1).

The LSP setup request $(P A T H)$ carries the identifiers of the parent session and the LSP, together with the parameters that describe the requested LSP. LSP is accepted by the downstream router - proxy $\mathrm{B}$, which generates reservation message (RESV). Proxy routers $\mathrm{A}$ and $\mathrm{B}$ generate appropriate management information to switches 1 and 6 , respectively. Program XC denotes command to perform cross-connection in the data plane.

\section{PERformance ANALYSIS}

Two realistic regional transport networks, namely NET1 and NET2, have been studied, as illustrated in Fig. 3.

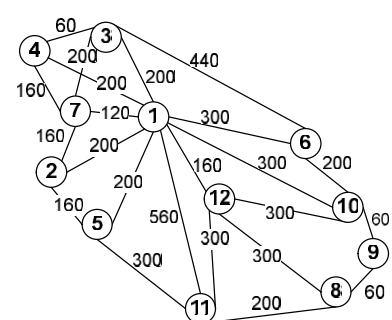

(a)

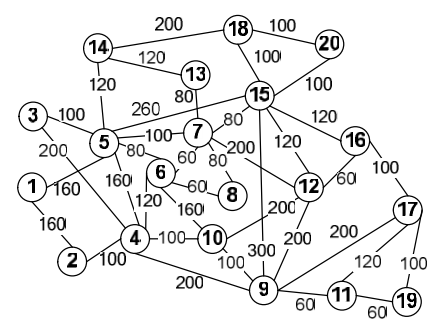

(b)
Fig. 3. The investigated topologies: (a) NET1 and (b) NET2. The number in the link shows the physical distance in kilometres.

Let $\mathrm{P}_{\max }$ be 0.5 for both networks. Using (1), we calculate $\quad \bar{k}_{\mathrm{NET} 1}=3.83$ and $\bar{k}_{\mathrm{NET} 2}=3.80$, and set $k_{\mathrm{T}, \mathrm{NET} 1}=k_{\mathrm{T}, \mathrm{NET} 2}=4$. Further, from (2) and (3) we obtain $p_{\mathrm{IF}, \mathrm{NET} 1}=0.348$ and $p_{\mathrm{IF}, \mathrm{NET} 2}=0.447$. Application of the proposed method to NET1 and NET2 is depicted in Fig. 4.

The three control plane configurations have been simulated and compared for each network, namely the IFOB, the OF, and the hybrid configuration. Simulations have been carried out using the network simulator NS2 [9] and its associated tools for the analysis of trace files - Trace Graph and NS2 Trace Visual Analyser.
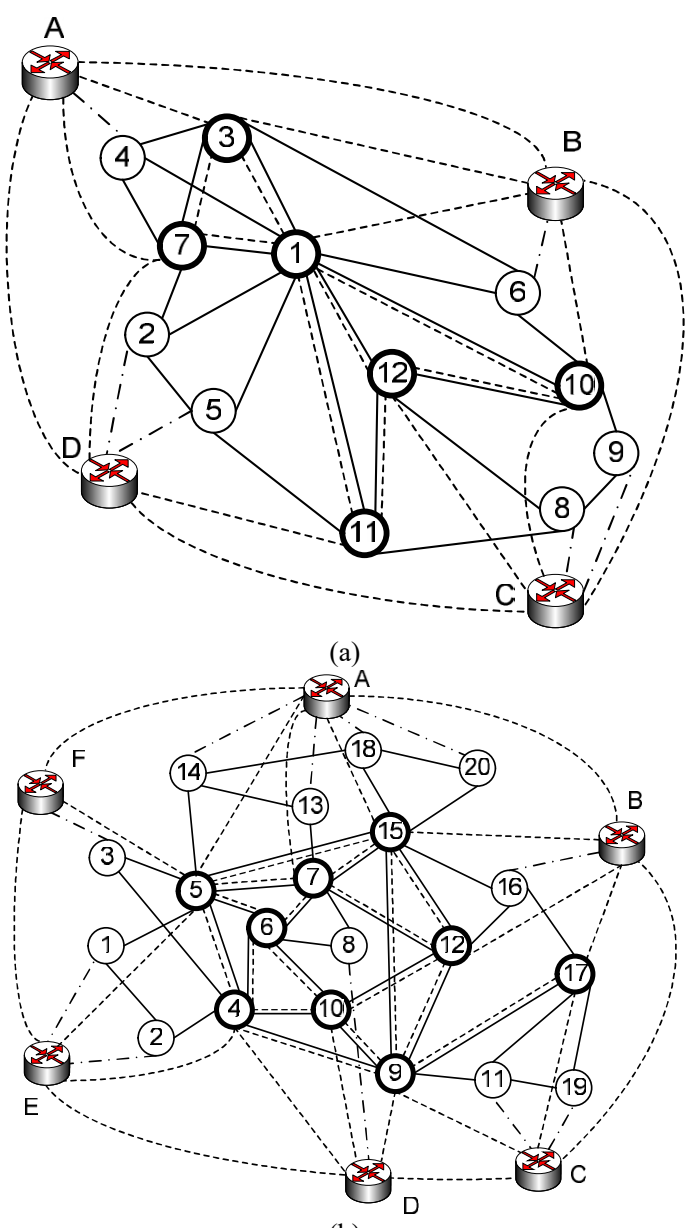

(b)

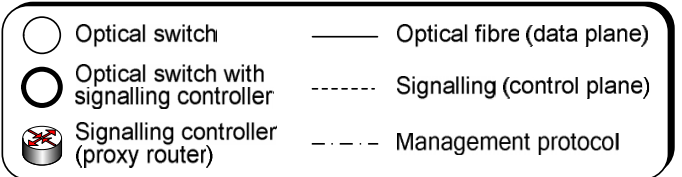

Fig. 4. Application of the proposed method: (a) NET1 and (b) NET2

In the IFOB configuration, signalling controllers are implemented in data switches. Link propagation delays are calculated as $t_{p}=d / v$, where $d$ represents physical distance (as shown in Fig. 3) and $v$ is the light propagation speed through fibre $\left(2 \cdot 10^{8} \mathrm{~m} / \mathrm{s}\right)$. Packet processing time at the signalling controller is $1 \mathrm{~ms}$ [10].

In the OF configuration, each switch is connected to the IP network, e.g., via Ethernet interface. The reserved bandwidth for signalling traffic on each link is $20 \mathrm{Mb}$. Delay requirements from the ITU-T Recommendation Y.1542 [11] have been satisfied, i.e., one-way packet transfer delay over regional domain should not exceed $15 \mathrm{~ms}$. We assume that average hop-by-hop delay is $2.5 \mathrm{~ms}$ (it includes propagation delay, queuing delay and packet processing time).

Hybrid configuration assumes control plane networks from Fig. 4. NET1 control plane consists of 6 built-in controllers (nodes 1, 3, 7, 10, 11 and 12) and 4 proxy routers (A - D). NET2 control plane consists of 9 built-in controllers (nodes $4,5,6,7,9,10,12,15$ and 17) and 6 proxy routers 
(A - F). Average hop-by-hop delays between routers and between router and signalling controller are $2.5 \mathrm{~ms}$ (including propagation delay, queuing delay and packet processing time, as in the case of the OF method).

Signalling protocol has been simulated by a single successful request-response transaction, with messages of 1000 bytes. Such a model corresponds to the exchange of RSVP-TE messages PATH and RESV. Clearly, the obtained results can easily be generalized (by multiplication) to the cases with multiple requests and responses. We suppose error-free and loss-free transmission of signalling traffic. Signalling messages are routed over the shortest paths (paths with the smallest number of hops if link costs are equal).

Suppose that one bidirectional LSP between each pair of nodes is established at the same time. Hence, the total number of simultaneously established paths is $S=N(N-1) / 2$. For the observed networks, we obtain $S_{\mathrm{NET} 1}=66$ and $S_{\mathrm{NET} 2}=190$.

Assume that $m_{i, j}, \quad(i, j=1,2, \ldots, N, i<j)$ is the overall number of nodes that constitute the bidirectional path between nodes $i$ and $j$. The number of signalling messages in a single request-response transaction is $M_{i, j}=2\left(m_{i, j}-1\right)$.

If $S$ paths are being established simultaneously, the total number of signalling messages, $M$, is

$$
M=\sum_{i=1}^{N} \sum_{j=1}^{N} M_{i, j}=2 \sum_{i=1}^{N} \sum_{j=1}^{N}\left(m_{i, j}-1\right)
$$

where $i<j$. Analytical expressions for $M$ can be derived for basic network topologies [8]. For the observed networks, the total number of messages is obtained by simulation as follows: $M_{\mathrm{NET} 1}=238$ and $M_{\mathrm{NET} 2}=902$.

Average and maximum LSP setup times are presented in Table I, for $k_{\mathrm{T}}=4$. We can notice that setup times obtained with the hybrid method are close to the ones obtained using the IFOB. Figure 5 and Fig. 6 present histograms of LSP setup times for the three signalling transport methods in networks NET1 and NET2, respectively. Setup times with hybrid method are rather evenly distributed between minimum and maximum values, particularly in NET2.

Reduction of delay in the hybrid method compared to the OF method results from the following reasons: (1) the smaller number of routers reduces the overall processing times, and (2) certain number of LSPs, i.e., 15 LSPs in NET1 $(22.7 \%)$ and 32 LSPs in NET2 (16.8\%), are established as with the IFOB method (without proxies).

TABLE I. LSP SETUP TIMES.

\begin{tabular}{|c|c|c|c|c|}
\hline \multirow{2}{*}{ Method } & \multicolumn{2}{|c|}{ NET1 } & \multicolumn{2}{c|}{ NET2 } \\
\cline { 2 - 5 } & $\begin{array}{c}\text { Average } \\
\text { setup time }\end{array}$ & $\begin{array}{c}\text { Maximum } \\
\text { setup time }\end{array}$ & $\begin{array}{c}\text { Average } \\
\text { setup time }\end{array}$ & $\begin{array}{c}\text { Maximum } \\
\text { setup time }\end{array}$ \\
\hline IFOB & $8.02 \mathrm{~ms}$ & $14.10 \mathrm{~ms}$ & $8.26 \mathrm{~ms}$ & $16.82 \mathrm{~ms}$ \\
\hline OF & $10.60 \mathrm{~ms}$ & $18.30 \mathrm{~ms}$ & $14.28 \mathrm{~ms}$ & $29.92 \mathrm{~ms}$ \\
\hline $\begin{array}{c}\text { Hybrid, } \\
k \mathrm{~T}=4\end{array}$ & $8.54 \mathrm{~ms}$ & $13.60 \mathrm{~ms}$ & $9.02 \mathrm{~ms}$ & $17.50 \mathrm{~ms}$ \\
\hline
\end{tabular}

It should be noted that only node 1 in NET1 has degree higher than 4, and also only node 9 has degree less than 3 .
Therefore, the choice $k_{\mathrm{T}}=4$ is the proper solution for NET1 control plane.

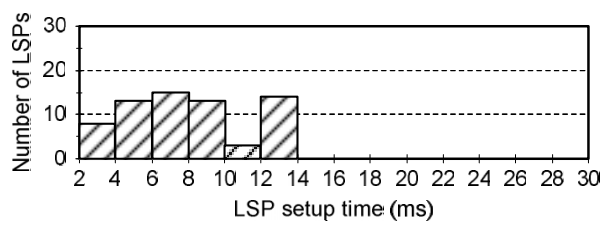

(a)

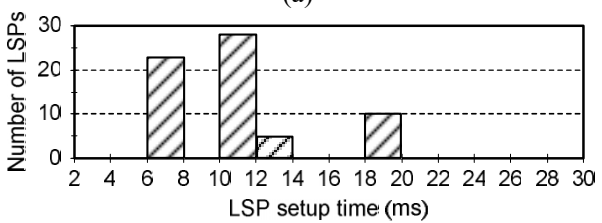

(b)

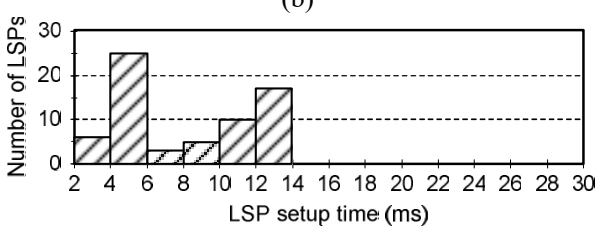

(c)

Fig. 5. Histograms of LSP setup times in NET1, with $k_{\mathrm{T}}=4, p_{\mathrm{IF}}=0.348$ : (a) IFOB method; (b) OF method; (c) hybrid method.

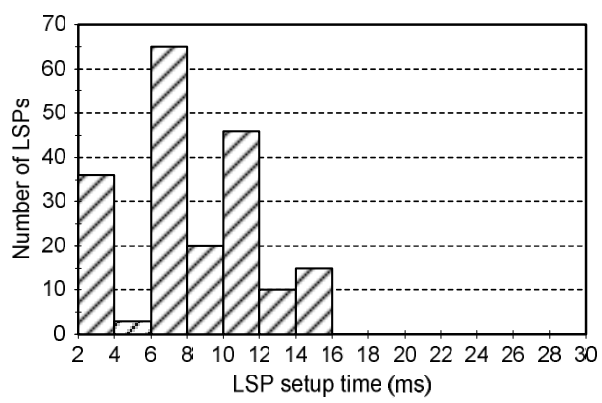

(a)

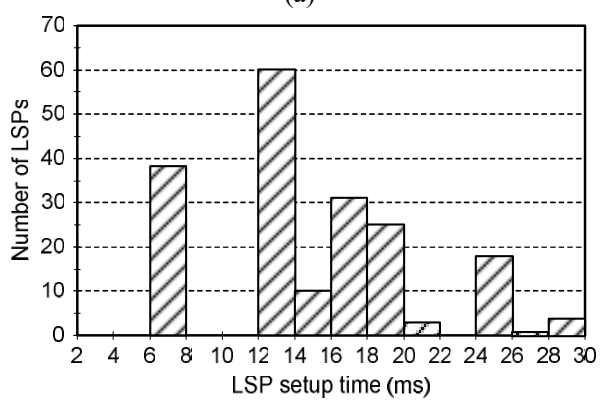

(b)

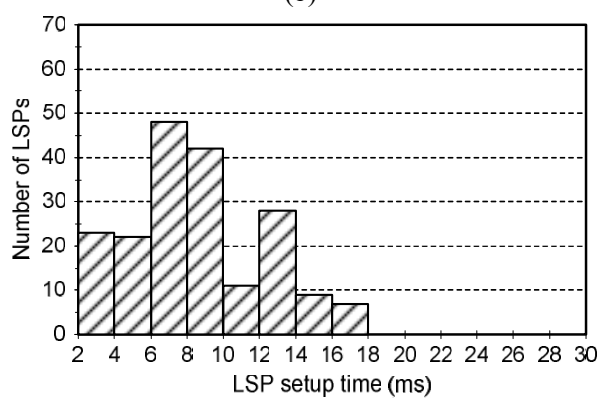

(c)

Fig. 6. Histograms of LSP setup times in NET2, $k_{\mathrm{T}}=4, p_{\mathrm{IF}}=0.447$ : (a) IFOB method; (b) OF method; (c) hybrid method.

The NET2 topology allows more options to select the threshold $k_{\mathrm{T}}$. Table II contains NET2 performance in terms of average and maximum LSP setup times, and the 
probability $p_{\mathrm{IF}}$ for higher values of the threshold, i.e., $k_{\mathrm{T}}=5,6$, and 7 . The obtained results show that the choice of $k_{\mathrm{T}}$ offers the designer the preference to reduce latency or to improve control plane network reliability.

TABLE II. NET2 PERFORMANCE FOR DIFFERENT VALUES OF THE NODE DEGREE THRESHOLD.

\begin{tabular}{|c|c|c|c|}
\hline kT & Average setup time & Maximum setup time & pIF \\
\hline 5 & $9.19 \mathrm{~ms}$ & $18.83 \mathrm{~ms}$ & 0.316 \\
\hline 6 & $11.98 \mathrm{~ms}$ & $23.50 \mathrm{~ms}$ & 0.158 \\
\hline 7 & $14.34 \mathrm{~ms}$ & $26.57 \mathrm{~ms}$ & 0.026 \\
\hline
\end{tabular}

The proposed hybrid method assumes a combination of a symmetrical control plane topology in the network segment where the IFOB is applied, and the asymmetrical topology in the network segment where the OF is applied. Hence, the overall control plane topology is asymmetrical and partialmesh. Based on the analysis and results from [5], restoration times are estimated to be between the ones achieved by the IFOB and OF methods, and are quite reasonable (below $100 \mathrm{~ms}$ even for continental wide area network topologies).

\section{APPLICABILITY to MUlTi-DOMAIN SCENARIOS}

Establishing LSPs over multiple domains poses a number of challenges for the control plane design. The main issues refer to path computation and delay requirements. Separation between the control plane and the data plane causes that any division of each of these planes can create a domain [1]. In the ITU-T automatically switched optical network (ASON) model [12], each domain in carrier's network is allowed to have independent characteristics.

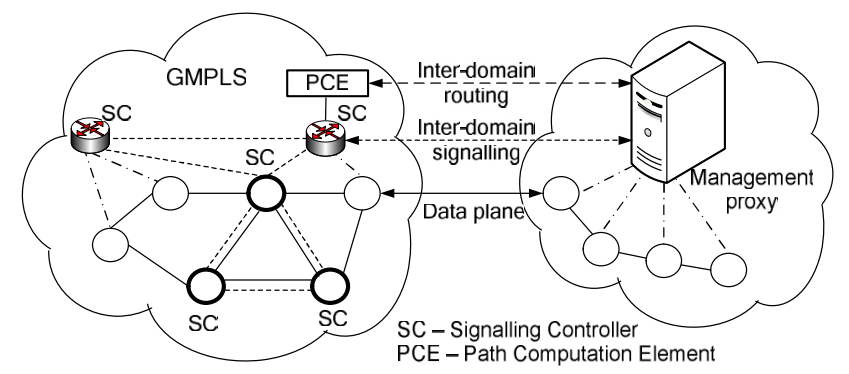

Fig. 7. An example of inter-domain scenario with the distributed (GMPLS) and centralized control planes.

Our proposed method for signalling transport can meet the end-to-end requirements for low latency, while supporting flexible integration with the algorithms for inter-domain path computing through PCEs. Moreover, it is fully applicable to scenarios in which some domains are managed by a distributed control plane (GMPLS) while others are managed by a centralized control plane such as OpenFlow [7], [13], [14], as illustrated in Fig. 7.

\section{CONCLUSIONS}

This paper presented a proposal for signalling transport method in GMPLS control plane networks. The method starts from the analysis of the data plane network topology in terms of node degrees and joint degree distribution, and further combines the benefits of the IFOB and $\mathrm{OF}$ approaches. The predefined node degree threshold determines the boundary between the IFOB and OF configurations in the control plane.

Our simulation results have indicated that LSP setup delays are close to the ones obtained with the IFOB method. Selection of the node degree threshold allows the designer to make a trade-off between signalling delay and the probability that a failure occurs on the link that carries both data and signalling in fibre. Such links should exist only between highly connected nodes and should be properly protected.

The proposed hybrid method is applicable to different multi-domain scenarios due to low latency, inherited from the IFOB approach, and high flexibility to integrate with the control plane network intelligence, benefited from the $\mathrm{OF}$ approach.

\section{REFERENCES}

[1] A. Farrel, I. Bryskin, GMPLS Architecture and Applications. Morgan Kaufmann Series in Networking, Elsevier, 2006, ch. 4 and ch. 7.

[2] L. Berger (Ed.), Generalized Multi-Protocol Label Switching (GMPLS) Signaling Resource Reservation Protocol - Traffic Engineering (RSVP-TE) Extensions, IETF RFC 3473, 2003.

[3] A. Takacs, L. Berger, D. Caviglia et al., GMPLS Asymmetric Bandwidth Bidirectional Label Switched Paths (LSPS), IETF RFC 6387, 2011.

[4] J. Perello, L. Velasco, F. Agraz, et al., "A comparison of in-fiber and out-of-fiber GMPLS-based control plane configurations: Benefits, drawbacks and solutions", in Proc. 10th Ann. Int. Conf. on Transparent Optical Networks, Athens, 2008, vol. 3, pp. 101-104 [Online]. Available: http://dx.doi.org/10.1109/ICTON.2008.4598665

[5] M. Ruiz, J. Perello, L. Velasco, et al., "GMPLS control plane network design with resilience guarantees", Journal of Lightwave Technology, vol. 29, no. 1, pp. 37-47, 2011. [Online]. Available: http://dx.doi.org/10.1109/JLT.2010.2090648

[6] M. Veeraraghavan, T. Li, "Signaling transport options in GMPLS networks: In-band or out-of-band", in Proc. 16th Int. Conf. on Computer Communications and Networks, Honolulu, Hawaii, 2007 , pp. 503-509. [Online]. Available: http://dx.doi.org/10.1109/ ICCCN.2007.4317869

[7] L. Y. Ong, E. Roch, S. Shew, A. Smith, "New technologies and directions for the optical control plane", Journal of Lightwave Technology, vol. 30, no. 4, pp. 537-547, 2012. [Online]. Available: http://dx.doi.org/10.1109/JLT.2011.2175364

[8] M. Stojanovic, V. Acimovic-Raspopovic, "Dynamic inter-domain IP QoS negotiation models: Performance analysis and comparison", in Proc. 7th Int. Conf. on Telecommunications in Modern Satellite, Cable and Broadcasting Services, Nis, Serbia, 2005, vol. 2, pp. 348 351. [Online]. Available: http://dx.doi.org/10.1109/telsks.2005. 1572125

[9] The Network Simulator NS2 and Network Animator NAM. [Online] Available: http://www.isi.edu/nsnam

[10] R. Ghimire, S. Mohan, M. Leary, T. Tidwell, "A concurrent two-layer restoration scheme for GMPLS WDM networks", Optical Switching and Networking, vol. 6, no. 4, pp. 268-275, 2009. [Online] Available: http://dx.doi.org/10.1016/j.osn.2009.08.005

[11] Framework for Achieving End-to-End IP Performance Objectives. ITU-T Recommendation Y.1542, 2010.

[12] Architecture for the Automatically Switched Optical Network. ITU-T Recommendation G.8080/Y.1304, 2012.

[13] P. Bhaumik, S. Zhang, P. Chowdhury, et al., "Software-defined optical networks (SDONs): a survey", Photonic Network Communications, vol. 28, no. 1, pp. 4-18, 2014. [Online]. Available: http://dx.doi.org/10.1007/s11107-014-0451-5

[14] S. Azodolmolky, R. Nejabati, E. Escalona, et al., "Integrated OpenFlow-GMPLS control plane: an overlay model for software defined packet over optical networks", Optics Express, vol. 19, no. 26, pp. 421-428, 2011. [Online]. Available: http://dx.doi.org/10.1364/OE.19.00B421 